\title{
80 MHz RADIOHELIOGRAPH EVIDENCE ON MOVING TYPE IV BURSTS AND CORONAL MAGNETIC FIELDS
}

\author{
S. F. S MERD \\ Division of Radiophysics, C.S.I.R.O., Sydney, Australia \\ and \\ G. A. DULK \\ Boulder, Colo., U.S.A.
}

Division of Radiophysics, C.S.I.R.O. and Department of Astro-Geophysics, University of Colorado,

\begin{abstract}
The characteristics of 12 moving type IV bursts observed with the $80 \mathrm{MHz}$ radioheliograph at the Culgoora Observatory between February 1968 and April 1970 are summarized.

Three classes of moving sources can be recognized; they are described as: (1) Expanding arch; (2) Advancing front; (3) Isolated source.

The first class has been identified (Wild, 1969) with the expansion of a magnetic arch or loop; the second class is here identified with an advancing MHD disturbance which may accelerate the radiating electrons in situ when moving at greater than Alfvén speed; the third with solar ejecta in the form of magnetized plasma clouds, or plasmoids. In all cases the radiation mechanism is probably synchrotron radiation from mildly relativistic electrons; energies in the range $\sim 0.1$ to $\sim 1 \mathrm{MeV}$ could account for the observed strong circular polarizations.

With an expanding magnetic arch, source and magnetic-field movement are inseparable; the field remains a closed loop throughout the event. The MHD front probably moves largely along and the plasmoids between the open magnetic-field lines of unipolar regions or helmet structures. In the latter case it is the internal magnetic field - possibly toroidal - of the moving plasmoid that determines the polarization of the synchrotron radiation. A preliminary comparison of moving type IV sources with Newkirk-Altschuler maps of coronal magnetic fields shows suitably located closed loops for 2 events identified as expanding magnetic arches and unipolar open field lines along the path of a moving source identified as a plasmoid.
\end{abstract}

\section{Introduction}

Type IV bursts were first recognized and classified as a separate type by Boischot (1957), largely on the basis of the long-lasting, smooth character of their radiation and the substantial movement of the burst source as observed with the $169 \mathrm{MHz}$ interferometer at Nançay. Subsequently, the type IV label was applied to all long-lasting, fairly smooth and wide-band continua at all wavelengths, even though most such radiation came from stationary sources. To distinguish the original type IV burst from other metre-wave bursts with similar spectral characteristics, Weiss (1963) introduced the terms 'moving' and 'stationary' type IV bursts. Characteristically, the moving type IV burst occurs at metre and longer wavelengths where it is probably the rarest type of burst. Wild et al. (1959) using swept-frequency interferometry showed that the radiation at all frequencies in the range $40-70 \mathrm{MHz}$ came from the same source region, as would be expected on Boischot and Denisse's (1957) hypothesis that synchrotron radiation is emitted by a moving source.

In Section 2 of this paper, we summarize the $80 \mathrm{MHz}$ radioheliograph observations of 12 moving type IV bursts observed in the period February 1968 to April 1970. 
Three bursts, which we consider to be representative of three phenomenologically different classes of moving type IV bursts, are described in greater detail. In Section 3, we discuss the physical implications of the observed properties of the bursts; this leads to a physical classification of moving type IV bursts. In Section 4 we relate the observed and derived characteristics of the bursts to the magnetic-field structure in the corona.

\section{The Observations}

\subsection{General}

Table I summarizes the characteristics of 12 moving type IV bursts and their association with other radio and optical activity on the Sun. Some of the parameters in Table I result directly from the radioheliograph observations; others are derived, using an additional assumption or supplementary data. For six events the derived distances and speeds are given by bracketed values in the Table. Of these, four events (1968 Feb. 25, 1969 Mar. 1-2, 1969 Oct. 10, 1970 Mar. 21) are 'limb events' in which the associated flare or eruptive prominence was located close to the limb and hence the observed distances were probably within $10 \%$ of the actual distances. Two events (1968 Nov. 22, 1970 Apr. 29) were associated with flares at intermediate longitudes and no supple-
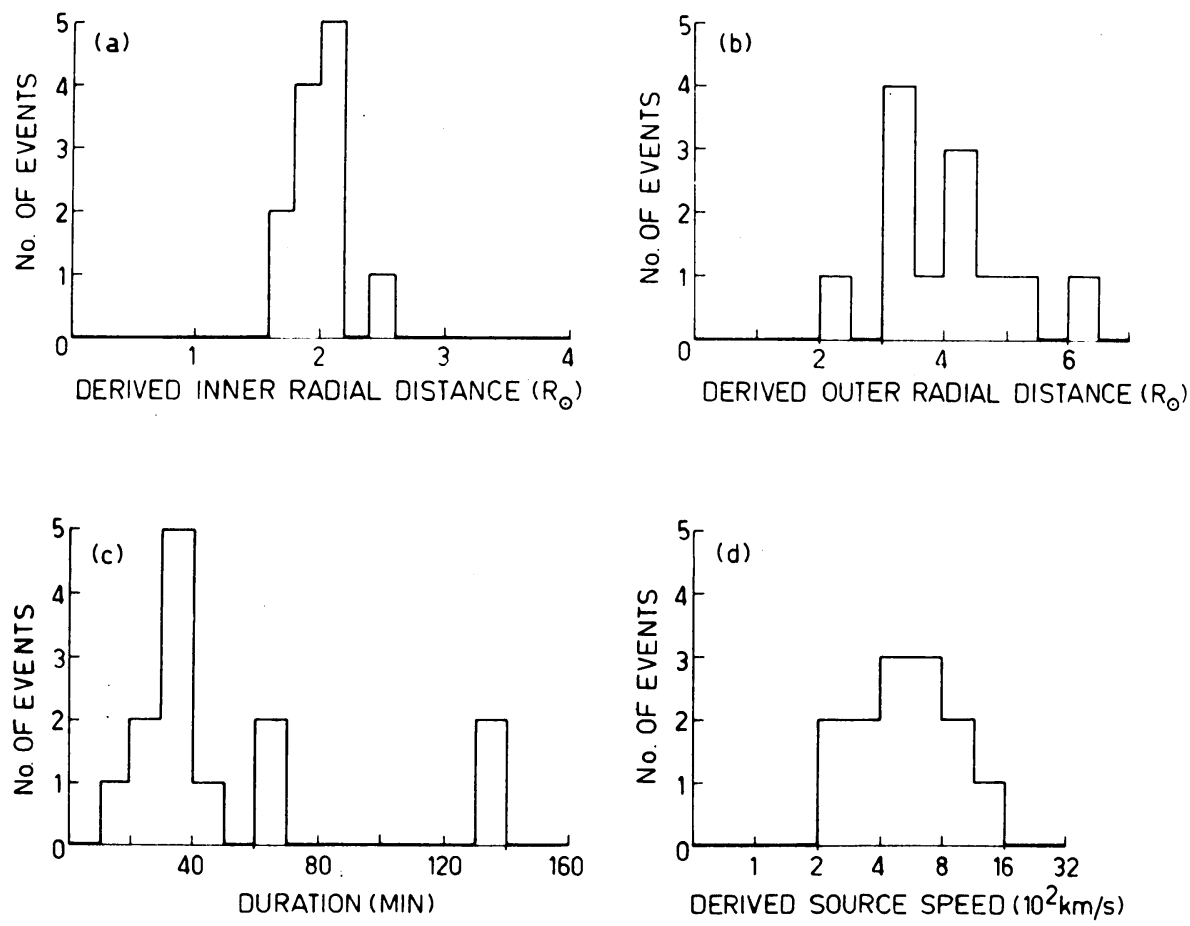

Fig. 1. Histograms of the derived inner (a) and outer (b) radial distances, duration (c), and derived source speed (d) for the 12 moving type IV bursts observed with the $80 \mathrm{MHz}$ radioheliograph between February 1968 and April 1970. 
TABLE

Characteristics of the twelve moving type-IV bursts observed with

\begin{tabular}{|c|c|c|c|c|c|c|c|c|c|c|c|c|}
\hline \multirow[t]{3}{*}{ Date } & \multicolumn{2}{|c|}{ Time (UT) of } & \multicolumn{2}{|l|}{ Associated events } & \multicolumn{4}{|c|}{ Distances form centre $(R \odot)$} & \multicolumn{4}{|l|}{ Velocity } \\
\hline & Start & Finish & \multirow[t]{2}{*}{ Radio } & \multirow[t]{2}{*}{ Optical } & \multicolumn{2}{|c|}{ Projected } & \multicolumn{2}{|c|}{ Derived } & \multicolumn{2}{|c|}{ Projected } & \multicolumn{2}{|l|}{ Derived } \\
\hline & h $\mathrm{m}$ & h $\mathrm{m}$ & & & First & Last & First & Last & $\begin{array}{l}\text { Speed } \\
(\mathrm{km} / \mathrm{s})\end{array}$ & $\begin{array}{l}\text { PA } \\
\left({ }^{\circ}\right)\end{array}$ & $\begin{array}{l}\text { Speed } \\
(\mathrm{km} / \mathrm{s})\end{array}$ & $\begin{array}{l}\text { Angle } \\
\text { from line } \\
\text { of sight } \\
\left(^{\circ}\right)\end{array}$ \\
\hline 1968 Feb. 25 & 0405 & 0430 & $\begin{array}{l}\text { III, II (+ II?) } \\
\mu \text {-burst (C) }\end{array}$ & $\begin{array}{l}1 B, 03^{\mathrm{h}} 48^{\mathrm{m}} \\
\text { S30, W17; } \\
\text { EPL }\end{array}$ & 1.6 & 2.0 & $(1.6)$ & $(2.0)$ & 400 & 120 & (400) & (90) \\
\hline 1968 Sep. 4 & 0059 & 0122 & $\begin{array}{l}\text { III, } 2 \text { II's, IVS } \\
\mu \text {-burst }(C)\end{array}$ & $\begin{array}{l}1 \mathrm{~N}, 00^{\mathrm{h}} 28^{\mathrm{m}} \\
\mathrm{N} 13, \mathrm{~W} 14 ; \\
\mathrm{DF}\end{array}$ & 0.6 & 1.4 & 2.5 & 4.0 & 500 & 90 & 760 & 41 \\
\hline 1968 Oct. 23-24 & 0006 & 0020 & $\begin{array}{l}\text { III, } 2 \text { II's IVS? } \\
\mu \text {-burst }(C)\end{array}$ & $\begin{array}{l}2 B, 23^{\mathrm{h}} 56^{\mathrm{m}} \\
\text { S12, E59; } \\
\text { ADF }\end{array}$ & 1.5 & 2.9 & 2.0 & 3.6 & 1200 & $\begin{array}{l}50-140 \\
\text { Max.displ. } \\
105\end{array}$ & (1400) & (57) \\
\hline 1968 Oct. 26 & 0120 & 0220 & $\begin{array}{l}\text { III, } 2 \text { II's, IVS } \\
\mu \text {-burst }(C)\end{array}$ & $\begin{array}{l}1 \mathrm{~N}, 01^{\mathrm{h}} 09^{\mathrm{m}} \\
\text { S20, E32; } \\
\text { ADF, DSD }\end{array}$ & 0.9 & 2.1 & 2.0 & 4.3 & 250 & 110 & $\begin{array}{l}\quad 480 \\
\text { (Identifie } \\
\text { speed of } \\
\text { type II) }\end{array}$ & $\begin{array}{l}30 \\
\text { fed with } \\
\text { fecond }\end{array}$ \\
\hline 1968 Nov. 22 & 0100 & 0145 & $\begin{array}{l}\text { IVS } \\
\mu \text {-burst }(C)\end{array}$ & $\begin{array}{l}\text { 1N, 00h56m } \\
\text { S16, E39; } \\
\text { APR }\end{array}$ & 1.2 & 2.0 & (1.9) & (3.2) & 210 & 115 & (334) & (39) \\
\hline 1969 Mar. 1-2 & $<2316$ & 0131 & $\begin{array}{l}\text { III, II } \\
\mu \text {-burst }(C)\end{array}$ & $\begin{array}{l}1 \mathrm{~N}, 21^{\mathrm{h}} 04^{\mathrm{m}} \\
\text { N08, W89; } \\
\text { EPL } 21^{\mathrm{h}} 36^{\mathrm{m}}\end{array}$ & 2.1 & 5.25 & $(2.1)$ & $(5.3)$ & 270 & -90 & (270) & (90) \\
\hline 1969 Mar. 21 & 0152 & 0228 & $\begin{array}{l}\text { III, } 3 \text { II's, IVS } \\
\mu \text {-burst }(C)\end{array}$ & $\begin{array}{l}2 \mathrm{~N}, 01^{\mathrm{h}} 42^{\mathrm{m}} \\
\mathrm{N} 19, \mathrm{E} 16 ; \\
\text { ADF }\end{array}$ & 1.1 & 2.4 & 1.6 & 4.8 & 470 & $\begin{array}{l}+10 \text { to } \\
-25\end{array}$ & $\begin{array}{c}980 \\
\text { (Ident. W } \\
\text { of type I }\end{array}$ & $\begin{array}{l}30 \\
\text { vith speed } \\
\text { II bursts) }\end{array}$ \\
\hline 1969 Aug. 29-30 & 2330 & 0000 & $\begin{array}{l}\text { III, II, } 2 \text { IVS's } \\
\mu \text {-burst (S) }\end{array}$ & $\begin{array}{l}\text { 1B, } 23^{\mathrm{h} 04 \mathrm{~m}} \\
\text { N04, E38; } \\
\text { ADF }\end{array}$ & 1.1 & 1.8 & 2.0 & 3.2 & 370 & 140 & 480 & 50 \\
\hline 1969 Oct. 10 & 0348 & 0422 & $\begin{array}{l}\text { None } \\
\text { (Initiating event } \\
\text { behind Sun, } W \text {-h }\end{array}$ & $\begin{array}{l}\text { Unknown } \\
\text { probably } \\
\text { hemisphere) }\end{array}$ & 1.8 & 3.4 & $(1.8)$ & (3.4) & $650-$ & -135 & $(650)$ & (90) \\
\hline 1969 Dec. 17 & $\int 0050$ & & $\begin{array}{l}\text { III, IVS } \\
\mu \text {-burst }(S-C)\end{array}$ & $\begin{array}{l}2 \mathrm{~B}, 00^{\mathrm{h}} 31^{\mathrm{m}} \\
\mathrm{N} 09, \mathrm{E36}\end{array}$ & 1.2 & 1.4 & 1.7 & 2.0 & & $80 \rightarrow 40$ & $700 \approx$ & 45 \\
\hline & ( 0116 & 0220 & & & 1.2 & 1.7 & 1.9 & $3.3 ?$ & 150 & $70 \rightarrow 50$ & 260 & 35 \\
\hline 1970 Mar. 21 & 0112 & 0330 & $\begin{array}{l}\text { III, } 2 \text { II's, IVS } \\
\mu \text {-burst }(S-C)\end{array}$ & $\begin{array}{l}2 \mathrm{~N}, 00^{\mathrm{h}} 23^{\mathrm{m}} \\
\mathrm{N} 20, \mathrm{E} 69^{\prime} \\
\text { EPL } 00^{\mathrm{h}} 30^{\mathrm{m}}\end{array}$ & 1.9 & 6.0 & (1.9) & (6.0) & $\begin{array}{l}340 \\
\text { (For mo } \\
\text { tant frag }\end{array}$ & $\begin{array}{c}40 \\
\text { ost dis- } \\
\text { gment) }\end{array}$ & (290) & (90) \\
\hline 1970 Apr. 29 & 0100 & 0130 & $\begin{array}{l}\text { III, IVS? } \\
\mu \text {-burst }(S-C)\end{array}$ & $\begin{array}{l}2 \mathrm{~N}, 00^{\mathrm{h}} 47^{\mathrm{m}} \\
\text { S10, E46; } \\
\text { ADF }\end{array}$ & 1.4 & 3.0 & (2.1) & (4.2) & 580 & 85 & (820) & (46) \\
\hline
\end{tabular}

In the 'Associated Radio Events' $\mu$-burst is used to denote burst activity ( $S$-simple, $C$-complex) reported at $\mathrm{cm}$ and $\mathrm{dm}$ wavelengths. In the 'Associated Optical Events' filament and prominence activity is indicated by the abbreviations of the ESSA 'Preliminary Reports - SDFR', namely: APR - active prominence; EPL - eruptive prominence on the limb; ADF - active dark filament; DSD - dark surge on disk; DF is used to denote the disappearance of a filament. 
I the $80-\mathrm{MHz}$ radioheliograph, February 1968-April 1970

\begin{tabular}{llll}
\hline Source Strcuture & Descriptive Class & Remarks & Reference \\
Spatial & Polarization & &
\end{tabular}

Single, elliptical Unknown Isolated source

Single, elliptical

$40 \rightarrow>85 \%$, LH

Isolated source

$10-20 \% \mathrm{LH}$

$50 \rightarrow \lesssim 100 \% \mathrm{LH}$

Jet

$110^{\circ}$-arch

t

$$
50 \rightarrow \lesssim 100 \% \mathrm{LH}
$$

Three sources

Apex: unpolarized feet: opp.polarized

Expanding arch

$A: 0 \rightarrow>70 \%$ RH

$B: 0 \rightarrow 60 \% \mathrm{LH}$

Single $(\mathrm{A}) \rightarrow$
Double $(A, B) \rightarrow$

Single $(A)$

Three sources $\left(M_{1} M_{2}\right.$

$M_{3}$ ) within arch

$M_{1}$ : unpolarized,

$M_{2}: 20 \rightarrow 70 \% \mathbf{R H}$,

$M_{3}: \approx 15 \% \mathrm{LH}$

Inverted $U$

$0 \rightarrow 80 \%$

bi-polar

Single $\left(M_{1}\right) \rightarrow$

Double $\left(M_{1} M_{2}\right) \rightarrow$

$M_{1}: 0 \rightarrow \approx 50 \% \mathrm{LH}$

$M_{2}: 0 \rightarrow \approx 30 \% \mathrm{LH}$

$M_{3}: 40 \rightarrow>80 \% \mathrm{LH}$

Triple $\left(M_{1} M_{2} M_{3}\right)$

(Single

$0 \rightarrow 90 \%$ RH

$>90 \%$ LH

$30 \rightarrow>90 \%$ RH

Single $\rightarrow$

Four fragments

Single
$<10 \rightarrow>70 \%$ LH
Advancing front

Expanding arch (edge on?)

Isolated source

Expanding arch

Expanding arch

Isolated Source

Isolated source

Isolated source
Most movement late in life (after $04^{\mathrm{h}} 18^{\mathrm{m}}$ ); associated with triggered prominence eruption

Same speed and direction as second type II

Same speed and general direction as second type II

Accompanied for half-hour by weak, Stewart et al. (1970); RH polarized source moving from Stewart (1971) $\left(0.8 R \odot, 140^{\circ}\right)$ to $\left(1.1 R \odot, 160^{\circ}\right)$

Attributed to expanding magnetic arch

In direction of eruptive prominence, delayed $\approx 1^{\mathrm{h}}$

II and IV attributed to same shock wave (expanding magnetic arch?)

Bi-polar $\cap$-shaped apex accompanied by two stationary feet on disk

Initial movement southward; sources diverge while fading rapidly

$\{$ Peripheral movement; remained $j$ at end poirit for $\approx 1^{\text {h }}$

( Slightly curved path, $\approx 45^{\circ}$ to radial. Both sources expand while fading Centroid velocity $290 \mathrm{~km} / \mathrm{s}$; from $02^{\mathrm{h}} 30^{\mathrm{m}}$ source expands at $90 \mathrm{~km} / \mathrm{s}$ and fragments

Intermittent III bursts from $10 \mathrm{~m}$ before to end of event
Kai (1970)

Wild (1969)

Riddle (1970)

Wild et al. (1968)

Kai (1969a, b)

Stewart and Sheridan (1970)

Dulk (1970, 1971)

Smerd (1971)

Dulk et al. (1971)

Sheridan (1970)

Dulk and Altschuler (1971)

The 'Projected Direction' is given by the Position Angle measured eastward (+) and westward (-) from the north of the projected rotational axis of the Sun. In the 'Derived Distances from Centre' and in the 'Derived Speed and Direction' the bracketed values were derived on the assumption of radial propagation; a 'Derived Direction' of $\left(90^{\circ}\right)$ indicates that the event is considered a limb event. 
mentary evidence on their directions of motion was available; in these cases, the motion was assumed to be radial.

With due allowance for the uncertainty in the initial and final heights of a few events, Table I and Figure 1a show that characteristically the sources were first seen at a radial distance of about $2 R_{\odot}$. In a coronal model with twice the densities of Newkirk's (1961) active region (hereafter designated our standard density model), this initial height corresponds to the $44 \mathrm{MHz}$ plasma level, $0.4 R_{\odot}$ above the $80 \mathrm{MHz}$ plasma level. Similarly, Table I and Figure $1 \mathrm{~b}$ show that the final radial distance averages $3.9 R_{\odot}$, which is approximately the $20 \mathrm{MHz}$ plasma level.

The burst durations, shown in Figure 1c, average $51 \mathrm{~min}$. The sample suggests, but is too small to establish, that the distribution is bi-model. The two 'long-duration' events are also the events with the greatest outer radial distances.

Figure 1d shows that the average derived speed is $602 \mathrm{~km} / \mathrm{s}$. The two 'long-duration and distant' limb events were relatively slow moving, at about half the average speed ( 270 and $290 \mathrm{~km} / \mathrm{s}$ respectively). Figure 2 presents the same data as a comparison of burst duration and radial distance moved.

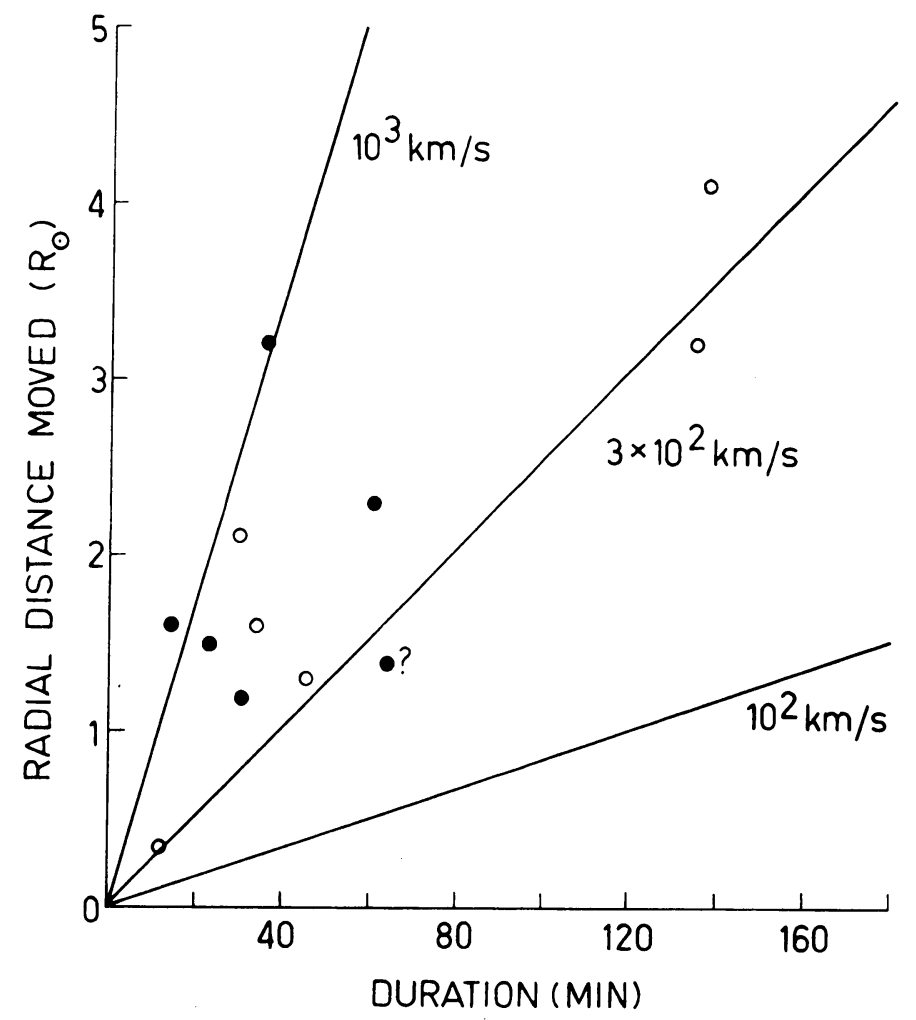

Fig. 2. The radial distance moved as a function of the duration for 12 moving type IV bursts. The filled circles refer to events for which the direction of motion was derived from supplementary data, the open circles to events where the motion was assumed to be radial. Three lines of constant speed are shown for comparison. 
At the time of the first event (Feb. 1968), polarization was not measured by the radioheliograph; of the other 11 events, 9 attained circular polarization greater than $70 \%$. In all these events the polarization increased with time, in some cases from initially zero polarization. In some events multiple sources existed from the beginning (1968 Nov. 22; 1969 Aug. 29-30) while in other events, a single source either fragmented into several components or new components appeared nearby (1969 Mar. 1-2, Mar. 21, Oct. 10; 1970 Mar. 21). In one case (1969 Mar. 1-2) there were two sources with opposite circular polarizations. In three cases, (1968 Nov. 22; 1969 Mar. 21 and 1969 Aug. 29) two oppositely polarized sources were accompanied by, or overlapped into, a third unpolarized source. In two cases (1969 Oct. 10; 1970 Mar. 21), the three or four moving sources all had the same sense of circular polarization.

The associated optical flares were not remarkable. The flare importance ranged from $1 \mathrm{~N}$ to $2 \mathrm{~B}, 6$ flares were of importance 1 and 5 of importance 2, 7 were of normal brightness and 4 were listed as bright. In one case, 1969 Oct. 10, the flare probably occurred beyond the west limb and was not observed.

The outstanding optical feature related to the bursts is the almost invariable association with active or disappearing filaments (disk events) and active or eruptive prominences (limb events).

Ten of the events were preceded by type III bursts and 8 by type II bursts, 6 of which were multiple type II bursts. The absence of type II observations in some of the remaining events may be linked with the much higher sensitivity of the radioheliograph compared with the Culgoora spectrograph. Many of the moving type IV events were quite weak $\left(\lesssim 10^{-21} \mathrm{Wm}^{-2} \mathrm{~Hz}^{-1}\right.$ ); indeed two of the events (1968 Nov. 22; 1969 Oct. 10) were not detected by the spectrograph. We conclude, as did Boischot and Denisse (1957), that there is a strong association between type II and moving type IV bursts. It may be significant that in several cases (e.g. 1968 Sept. 4, Oct. 23-24; 1969 Mar. 21) the moving type IV sources were associated with a slower-moving, second or third component of a multiple type II burst.

Following Wild (1970a), we distinguish three types of continuum emission associated with these events: (1) 'flare continuum', which appears soon after the flare starts, (2) moving type IV continuum, and (3) 'storm continuum' or the stationary type IV phase, which follows the moving type IV phase. Of the twelve events described here, at least 4 and possibly 7 included a flare continuum phase. Typically, the flare continuum comes from a large source $\left(\simeq 15^{\prime}\right.$ diam) and is unpolarized. Nine events included a storm continuum phase, typically with a small source $\left(\simeq 5^{\prime}\right.$ diam $)$ and strong circular polarization.

\subsection{INDIVIDUAL EVENTS}

In this section we describe four events in some detail; three illustrate fairly distinct classes of moving type IV bursts, while the fourth may fit either of two classes.

\section{Example 1: Isolated Source of 1970 March 21}

Sheridan (1970) reported a moving type IV source which moved outward to a radial 
distance of more than $6 R_{\odot}$. The event started at $00^{\mathrm{h}} 23^{\mathrm{m}}$ with a $2 \mathrm{~N}$ flare at N20, E69 and a spectacular eruptive prominence (Figure 3 ). The prominence material moved with a speed of about $260 \mathrm{~km} / \mathrm{s}$ at position angle (P.A.) $36^{\circ}$ and was last seen at a radial distance of $1.3 R_{\odot}$ at $00^{\mathrm{h}} 54^{\mathrm{m}}$. Approximately 20 min later the moving type IV source became visible at $1.9 R_{\odot}$ and at the same position angle, whence it moved

1970 MAR. 21
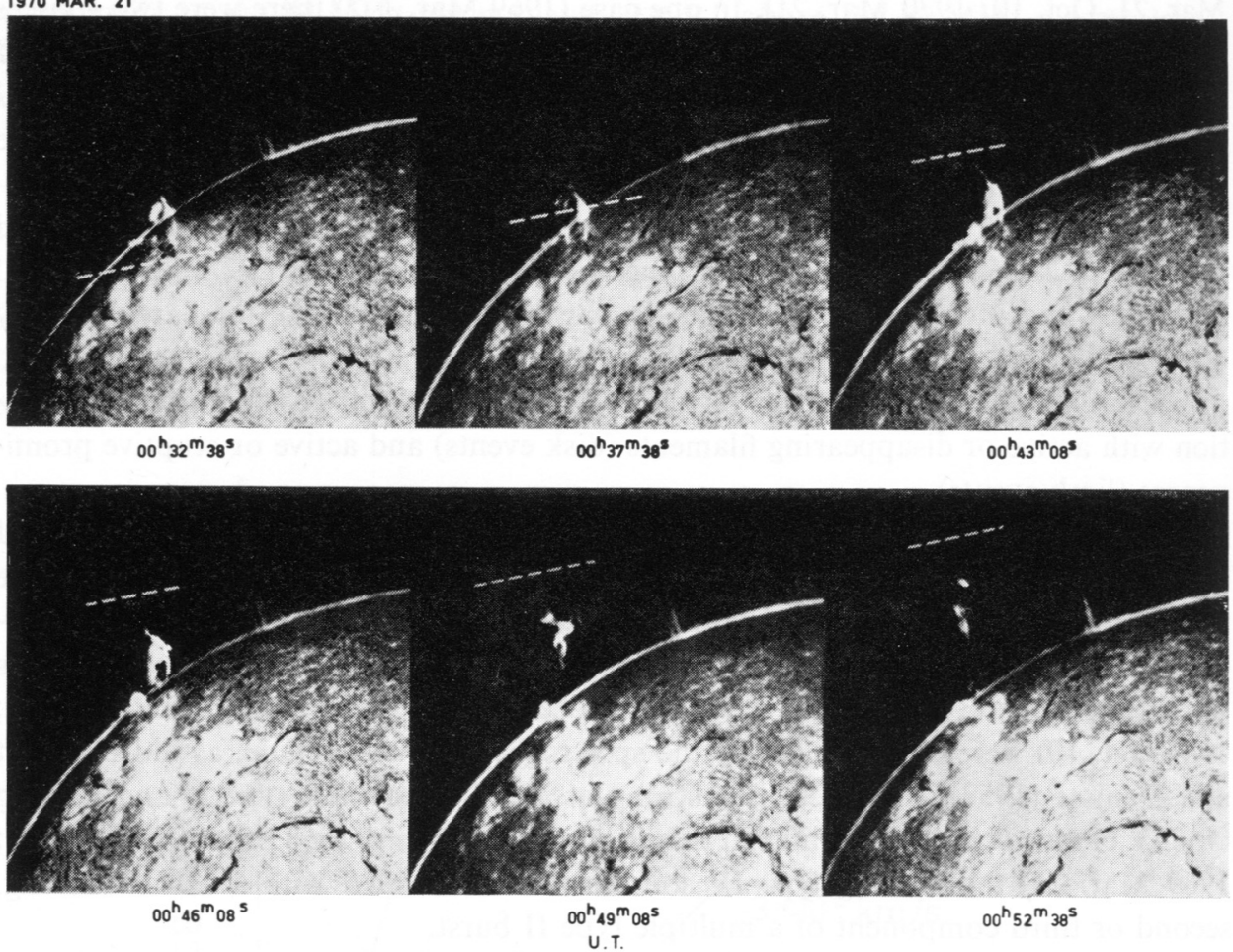

Fig. 3. H $\alpha$ filtergrams of a spectacular prominence eruption that preceded the far-moving type IV burst of 1970 March 21 . The broken lines move out from the apparent starting point (marked $X$ at the top left) at $290 \mathrm{~km} / \mathrm{s}$, the speed of the radio source (Sheridan, 1970).

outward at about $290 \mathrm{~km} / \mathrm{s}$ for about $90 \mathrm{~min}$. The evolution of the source is illustrated by the contour diagrams of Figure 4 . The radio source seen at $00^{\mathrm{h}} 55^{\mathrm{m}}$ ( 2 nd from top) is the early, stationary flare continuum. It is distinct from the moving source which first appeared in the same vicinity at about $01^{\mathrm{h}} 12^{\mathrm{m}}$. The moving source remained single and expanded slightly until $02^{\mathrm{h}} 30^{\mathrm{m}}$, at $3.7 R_{\odot}$, when it broke up. The four major fragments moved apart at about $90 \mathrm{~km} / \mathrm{s}$ while the centroid of the configuration continued outward at $290 \mathrm{~km} / \mathrm{s}$. During the outward progress the polarization increased from $30 \% \mathrm{RH}$ to more than $90 \% \mathrm{RH}$, with all fragments polarized to the same high degree and in the same sense. A storm continuum developed after $02^{\mathrm{h}} 00^{\mathrm{m}}$ and remained visible until $03^{\mathrm{h}} 30^{\mathrm{m}}$, again at a radial distance of about $1.9 R_{\odot}$. This source also showed RH polarization, which gradually increased from $30 \%$ to $70 \%$. 


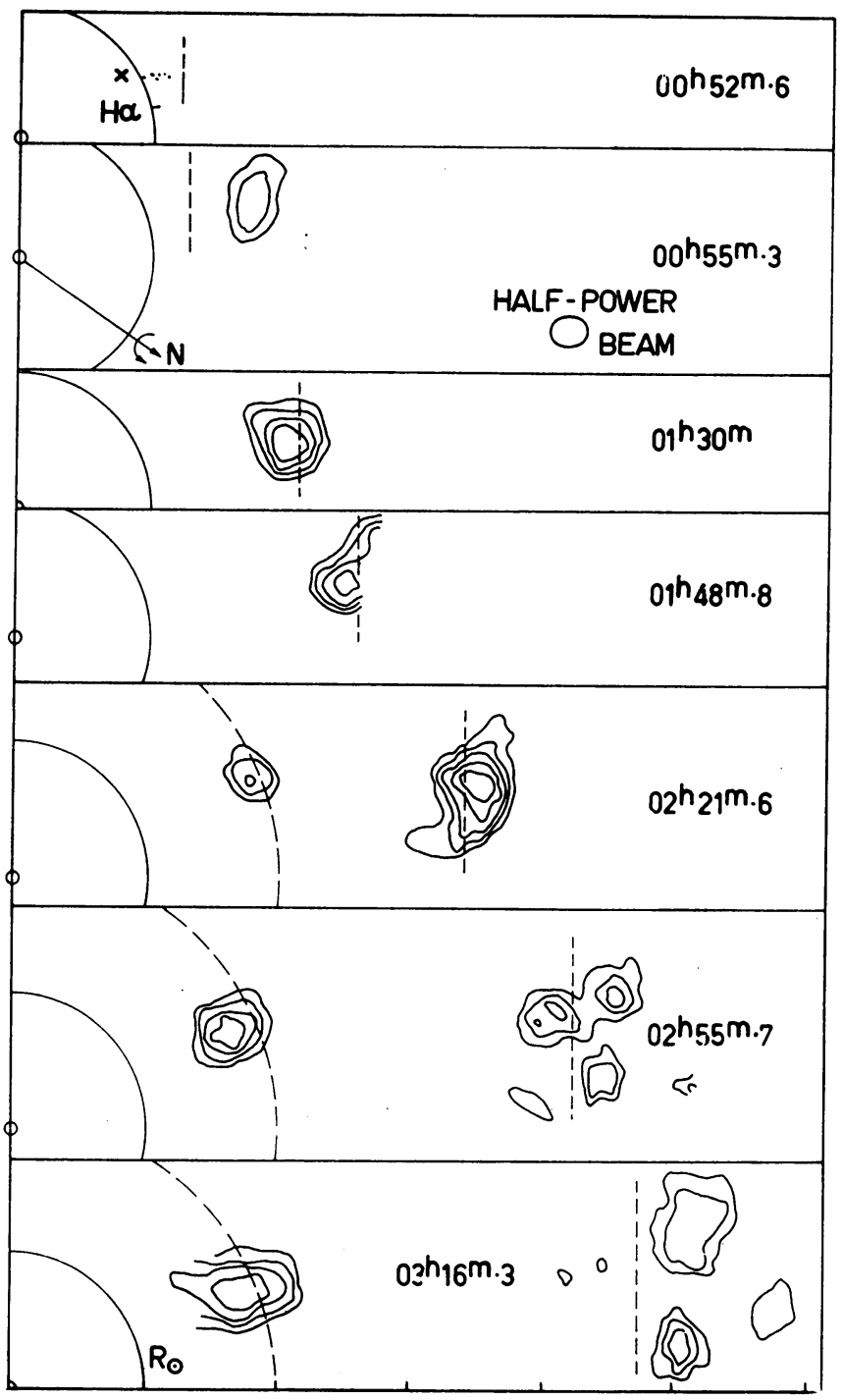

Fig. 4. $80 \mathrm{MHz}$ contours illustrating the outward movement and fragmentation of the 'isolated source' of March 21 (adjacent contours are in the ratio $\sqrt{ } \mathbf{2}: 1$ ). The broken lines denote a disturbance speed of $290 \mathrm{~km} / \mathrm{s}$. The top picture shows schematically a late phase of the eruption observed in $\mathrm{H} \alpha$ (see Figure 3) (Sheridan, 1970).

The spectrum showed that the type IV continuum was preceded by two type II bursts. The velocity of the second and stronger type II shock wave, deduced from the frequency drift and the standard density model, was $>1500 \mathrm{~km} / \mathrm{s}$. In view of the large and irreconcilable difference between the speeds of the type II shock front $(>1500 \mathrm{~km} / \mathrm{s})$ and of the moving type IV source ( $<300 \mathrm{~km} / \mathrm{s})$, it is clear that, in this case, the type II and the moving type IV bursts did not result from the same outward-moving disturbance. 
Example 2: Expanding Arch of 1968 November 22

The evolution of this event (Wild, 1969) is illustrated in Figure 5, which shows the development of an unmistakable arch structure. At times during the early phases, the arch became strongly circularly polarized, LH on the east side, RH on the west.

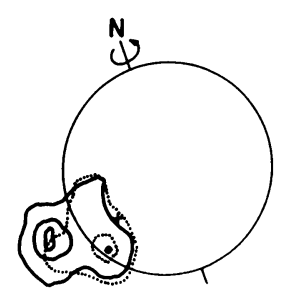

(a) $01^{h} 01^{m_{33} s}$

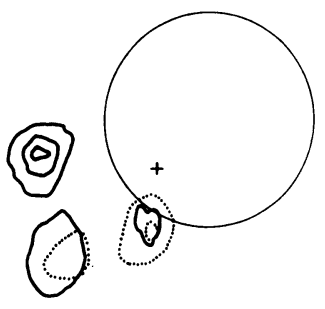

(d) $01^{\mathrm{h}} 23^{\mathrm{m}} 30^{\mathrm{s}}$

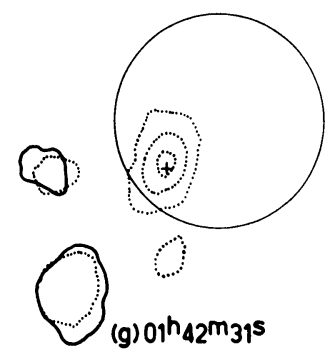

1968 NOVEMBER 22

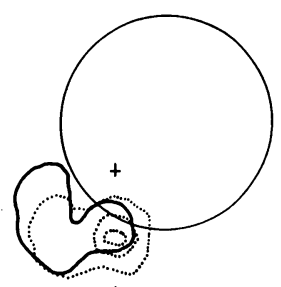

(b) $01 n_{13} m_{11} s$

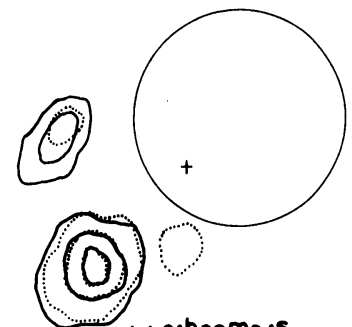

(e) $01^{h} 33^{m_{3} s}$

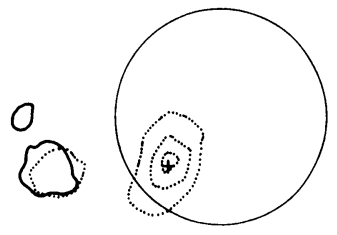

0

(h) $01^{h_{44} m_{34} s}$

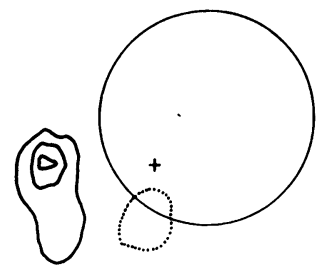

(c) $01^{h_{19} m_{02}}$

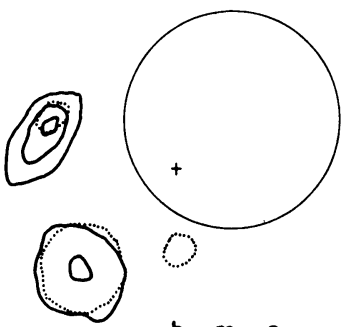

(f) $01^{\text {h }} 34^{m} m_{59}$

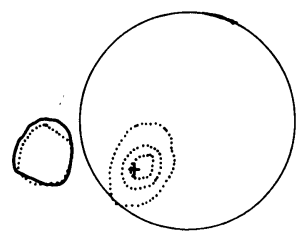

(i) $01 h_{51} m_{37} s$

Fig. 5. $80 \mathrm{MHz}$ contours illustrating the development at the indicated times of the 'expanding magnetic arch' of 1968 November 22. Full-line and broken-line contours represent LH and RH polarization respectively. The three contours represent 15,50 and $85 \%$ of peak brightness. The circles in this and later Figures indicate the optical disk of the Sun (After Wild, 1969).

As the arch expanded, the emission concentrated in three sources; two oppositely polarized sources near the feet of the arch and an unpolarized source (at times with oppositely polarized edges) at the top. During the development of the arch the projected rate of expansion decreased from about $300 \mathrm{~km} / \mathrm{s}$ to $100 \mathrm{~km} / \mathrm{s}$. The unpolarized source reached a projected distance of $2 R_{\odot}$ before the three sources began to fade. While the arch sources faded, a storm continuum, strongly RH polarized, appeared above the flare region. 
The spatial distribution and polarization of the sources strongly suggest an expanding magnetic arch (Wild, 1969), in which the unpolarized source resulted from synchrotron emission from a concentration of energetic electrons trapped in circular orbits near the top of the arch and the sources near the feet of the arch resulted from plasma radiation from electrons whose mirror points lay below the $80 \mathrm{MHz}$ plasma level.

\section{Example 3: Advancing Front of 1968 October 23-24}

Kai (1970) reported a complex event which was associated with a 2B-flare and filament activation. The radio spectrum showed numerous type III bursts, two type II bursts and a moving type IV burst, and a final phase which may be best described as a storm continuum. Figure 6 illustrates the various burst positions at different times during the event; we note that the type.II source and the type IV movement are in the same direction from the flare. All parts of the moving type IV front were slightly $(<20 \%)$ LH-polarized. The possible storm continuum, which settled down over the flare region near the end of the event, was $30-40 \%$ LH-polarized.

The derived velocities of the two II shock fronts were 3100 and $1400 \mathrm{~km} / \mathrm{s}$ respectively. The projected velocity of the advancing type IV front was $1200 \mathrm{~km} / \mathrm{s}$; correcting for a line-of-sight component (assuming radial propagation) the velocity becomes

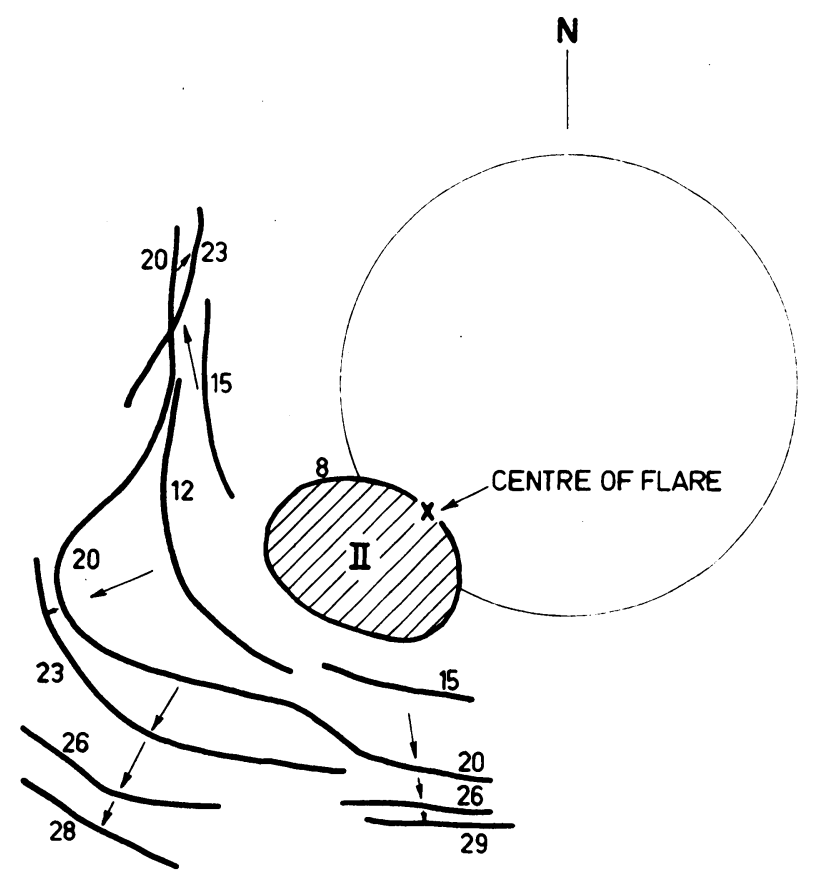

Fig. 6. Schematic diagram illustrating the evolution of the 'advancing front' of 1968 October 23-24. The thick lines represent the fronts along which radiation was concentrated. The numbers give the time in minutes from the start of the flare (Kai, 1970). 
$1400 \mathrm{~km} / \mathrm{s}$. Figure 7 illustrates the excellent agreement and continuity between the heights and speeds of the second type II burst and the movement of the type IV source. Of the twelve moving type IV bursts listed in Table I, this event shows the closest relationship between the type II and type IV bursts. It is unique for the large peripheral extent and the high speed of the outward-moving front.
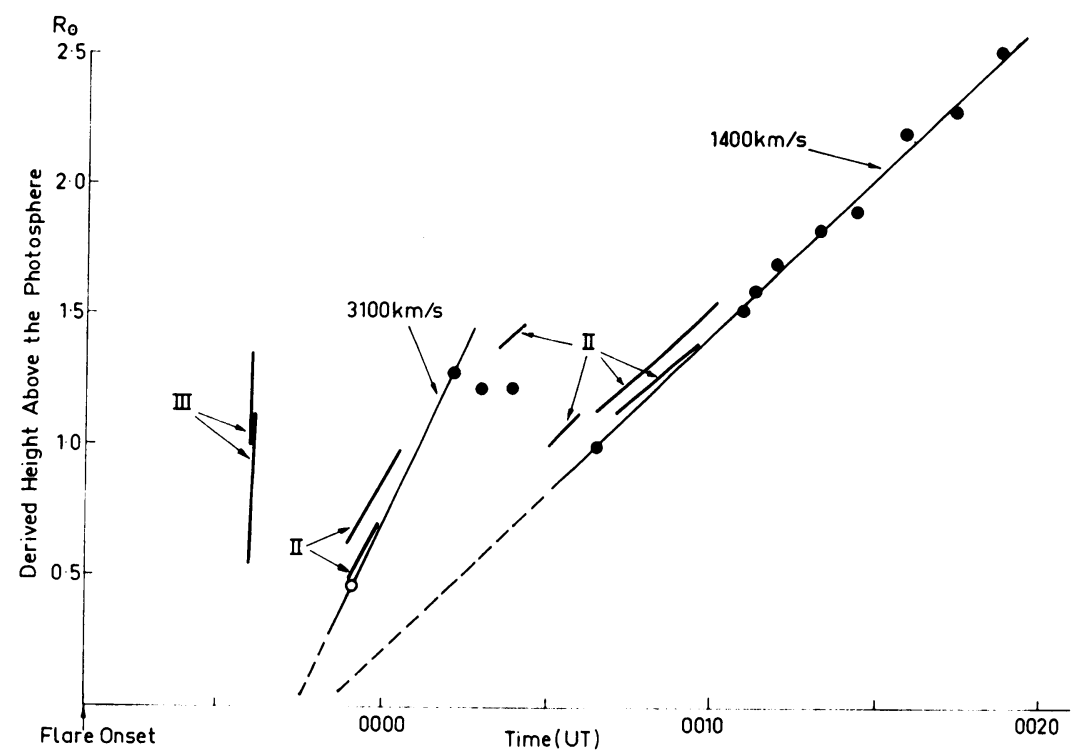

Fig. 7. Height-time plot for the type III and type II bursts and for the 'advancing front' type IV source of 1968 October 23-24. All heights were derived on the assumption that the sources lay radially above the flare. The type III and type II lines were derived from the dynamic spectra and the standard coronal model (see text); the open and filled circles were derived from the source positions observed with the radioheliograph. The filled circles beyond $1.5 R_{\odot}$ relate to the advancing front (Kai, 1970).

\section{Example 4: Expanding Arch or Advancing Front of 1969 March 21}

Stewart and Sheridan (1970) reported an outburst with multiple type II and moving IV bursts which combined some of the features of Examples 2 and 3 above. The type II bursts implied a disturbance moving outwards with a radial velocity of $980 \mathrm{~km} / \mathrm{s}$. When the type II disturbance reached a distance of $3 R_{\odot}$ from the Sun's centre (as derived from the spectrum), the source of the type IV burst started its outward movement at a projected speed of $470 \mathrm{~km} / \mathrm{s}$. The movement, the spatial structure and the polarization structure of this burst are illustrated by the contour diagrams of Figure 8 . The central part of the source remained unpolarized; the adjacent regions became $70 \% \mathrm{RH}$ and $20 \% \mathrm{LH}$ polarized respectively.

Stewart and Sheridan have interpreted this event as one in which type II and the type IV bursts originate in a common advancing shock front. By identifying the type II and type IV source velocities they deduced that the type IV source moved at an 
angle of $30 \%$ to the line of sight as illustrated in Figure 9. On this basis, the event is in the same class as Example 3.

Alternatively, this event may be interpreted as an expanding magnetic arch, such as that of Example 2, where the moving source is associated with the apex of the arch.
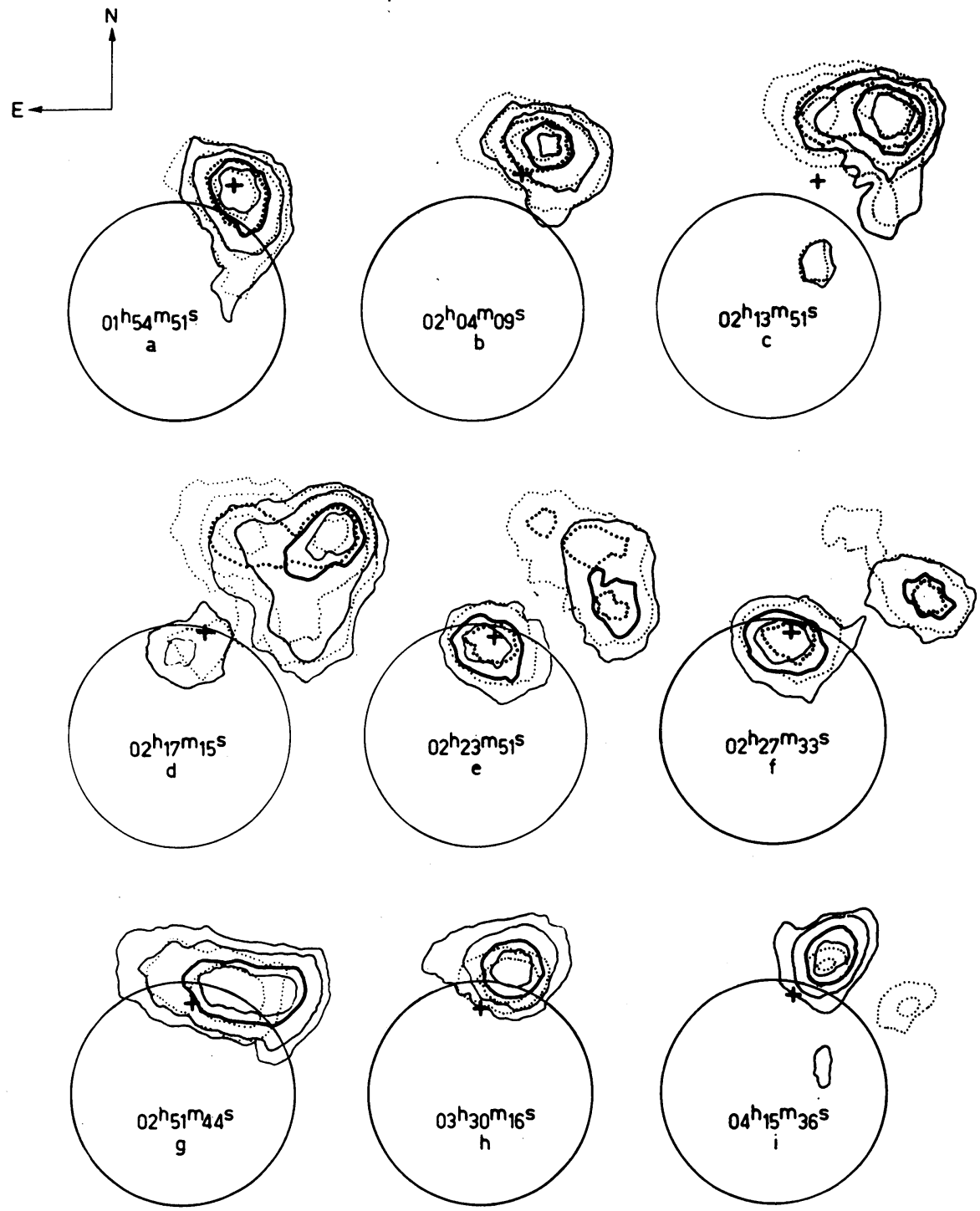

U.T. $02^{h}$

HALF - POWER BEAM

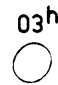

$0^{04^{h}}$

Fig. 8. $80 \mathrm{MHz}$ contours illustrating the advancing front or 'expanding arch' of $1969 \mathrm{March} 21$. Full-line and dotted-line contours represent $\mathbf{L H}$ and RH polarization respectively. Adjacent contours have a brightness ratio $\sqrt{ } \mathbf{2}: 1$. The crosses indicate centroid positions as measured with the $158 \mathrm{MHz}$ interferometer (Stewart and Sheridan, 1970). 


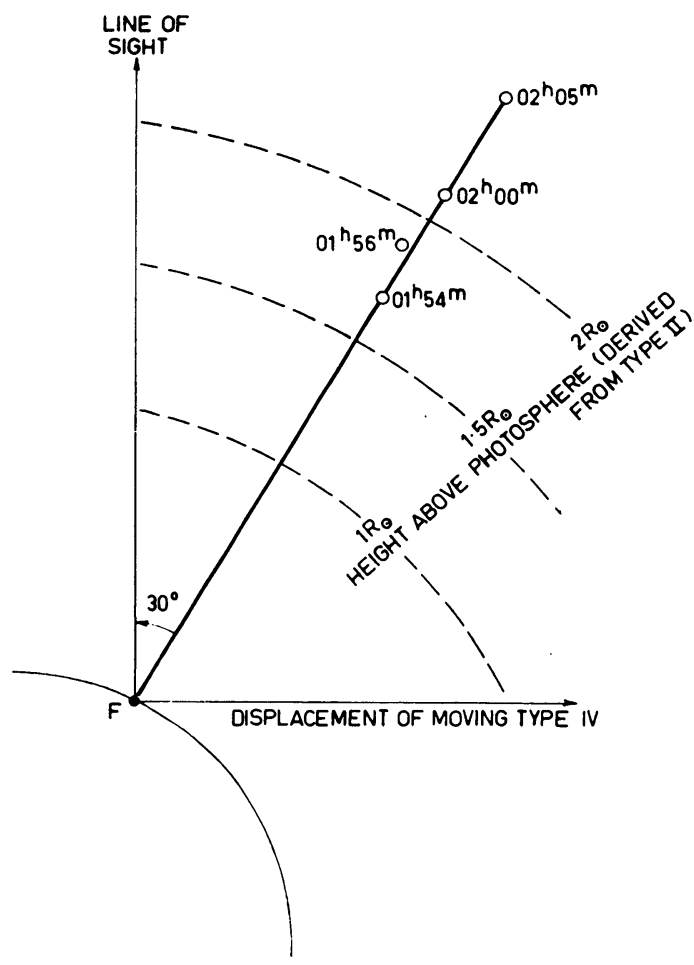

Fig. 9. The event of 1969 March 21 (see Figure 8) interpreted as an advancing front, with a common shock front exciting type II and moving type IV radiation. The speed of the moving type IV source is assumed to be identical with the shock speed derived from the spectrum of the last and most intense of three type II bursts. The required direction of the shock front is $30^{\circ}$ to the line of sight, as illustrated

(Stewart and Sheridan, 1970).

The source near the north point of the disk (Figure $8 d, e, f$ ) then represents one foot of the arch. In this interpretation the type II shock front is unrelated in speed or direction to the movement of the type IV source.

\section{Discussion}

\subsection{RADIATION MECHANISM}

We have seen that the type IV sources at $80 \mathrm{MHz}$ moved outwards from an average initial radial distance of $2 R_{\odot}$. In the standard density model (an active region) this corresponds to a plasma frequency $f_{p, A}=44 \mathrm{MHz}$, while in the background coronal plasma, $f_{p, B} \approx 15 \mathrm{MHz}$ (Van de Hulst, 1950). The ratios $\left(f / f_{p}\right)_{A}=1.8$ and $\left(f / f_{p}\right)_{B}=5.3$ are far too high for plasma radiation to account for the observed emissions. $80 \mathrm{MHz}$ plasma radiation could occur at a radial distance of $2 R_{\odot}$ only if the density were 6.7 times that in Newkirk's model of an active region.

Gyromagnetic radiation in the extraordinary $(x)$ mode and at the fundamental gyro-frequency, $f_{B}$, has been proposed (Fung, 1969) as a possible radiation mechanism. 
With this mechanism, the wide-band nature of the emission depends on a wide range of viewing angles. Narrow-band bursts should be common and large magnetic fields must necessarily be frozen into the moving plasma, e.g. $B=29 \mathrm{G}$ for $f_{B}=80 \mathrm{MHz}$. Another severe restriction is the requirement that $f_{p} / f_{B} \lesssim \frac{1}{3}$. Little is known about the value of $f_{p} / f_{B}$ or its changes in the moving source. However, as Fung (1969) pointed out, in the expanding source model used, for instance, by Zheleznyakov and Trakhtengerts (1965), $f_{p} / f_{B} \propto L^{1 / 2}$ - where $L$ is the linear dimension of the moving plasma. Therefore, gyroradiation, even if possible at the lowest levels, would soon cease as the moving cloud expanded.

Synchrotron radiation at the higher harmonics of the relativistic and Dopplershifted gyro-frequency has been invoked from the earliest interpretations of moving type IV bursts (Boischot and Denisse, 1957). The energy range of the radiating electrons is probably $100 \mathrm{keV}<E<1 \mathrm{MeV}$ because the bandwidth is often less than $100 \mathrm{MHz}$ and because the polarization is often strongly circular. A high energy cutoff of about $1 \mathrm{MeV}$ is also indicated by the condition $E>\frac{1}{2} f / f_{p}$ under which synchrotron radiation in a medium is thought to be suppressed (Ginzburg and Syrovatskii, 1965). The combination of suppressant medium and outward-moving source should make the onset of the burst drift from high to low frequencies in the spectrum; it has not been possible to check this feature for our bursts because the moving type IV component was usually weaker than the other spectral features with which it was intermingled.

\subsection{INTENSITY AND POLARIZATION CHANGES}

In Section 2 we found that the burst evolution usually involves increasing polarization and decreasing intensity. In an attempt to explain these features, Dulk (1970), using Wild's (1970b) much simplified formulae for the synchrotron emissivity of mildly-relativistic electrons, has evaluated the intensity and polarization of the radiation emitted by such electrons. The electron energy $(E)$ and pitch angle $(\phi)$ distributions were assumed to be of the form: $n_{E, \phi}(t)=n_{E}(t) n_{\phi}(t)$. The power-law energy distribution used was $n_{E}(t)=n_{E_{0}}(t)\left(E / E_{0}\right)^{-\alpha} \exp \left(-E / E_{1}\right)$, with $E_{1} \gg E_{0}$ (electrons with $E<E_{0}$, the low-energy cutoff, radiate negligible power; the high energy cutoff $E_{1}$ eliminates any small contribution from high-energy electrons with $\beta \gtrsim 0.95, E \gtrsim 1.2 \mathrm{MeV}$ where Wild's approximations are invalid). Two pitch-angle distributions were used: $n_{\phi}=$ constant, and $n_{\phi}=|\sin 2 \phi|$ (the latter corresponds to a deficiency of electrons at $\phi=0$ and $\pi / 2$ ). The results, plotted as a function of emission angle (with respect to the magnetic field), for the isotropic and anisotropic pitch-angle distributions are shown in Figures 10 a and $b$ respectively. The intensity peaks at $\theta=90^{\circ}$ for isotropic pitch angles and at $\theta \approx 60^{\circ}$ and $120^{\circ}$ for anisotropic pitch-angles. The intensity is widely distributed in angle, with half-power widths $\approx 80^{\circ}$ and $\approx 110^{\circ}$ respectively. With $f / f_{B}=10$ the degree of polarization at the half-power points is about $60 \%$ in the isotropic case and $75 \%$ in the anisotropic case. The circular polarization is right-handed for $\theta<\pi / 2$, left-handed for $\theta>\pi / 2$. The power and the degree of polarization decrease with increasing $f / f_{B}$, i.e. with increasing harmonic number. 


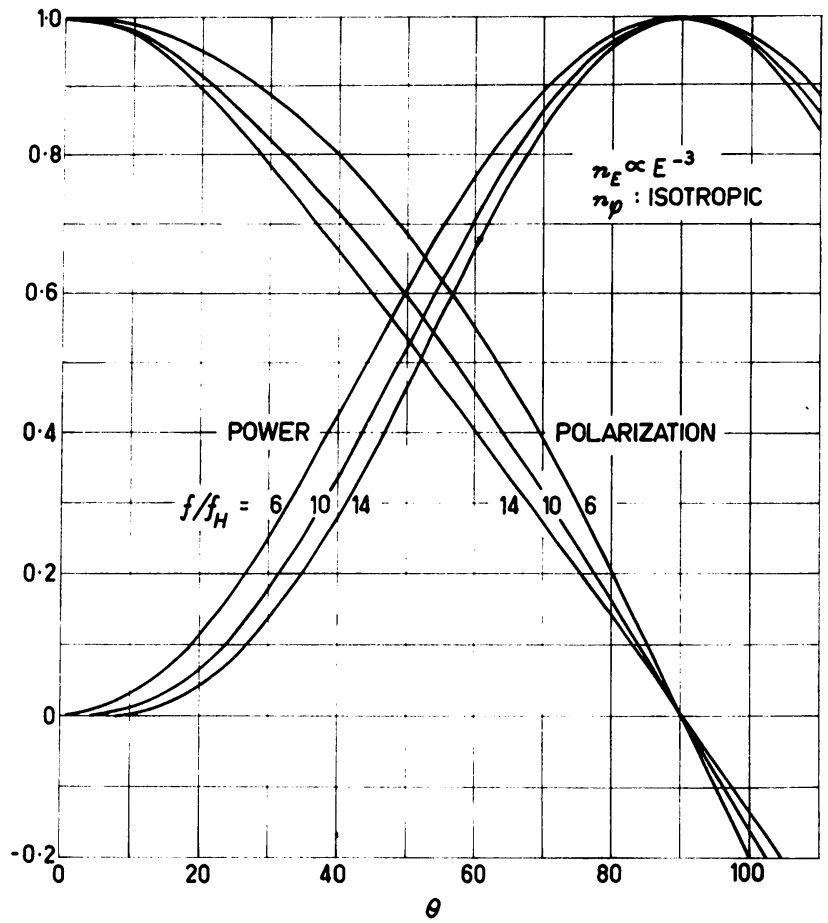

Fig. 10a.

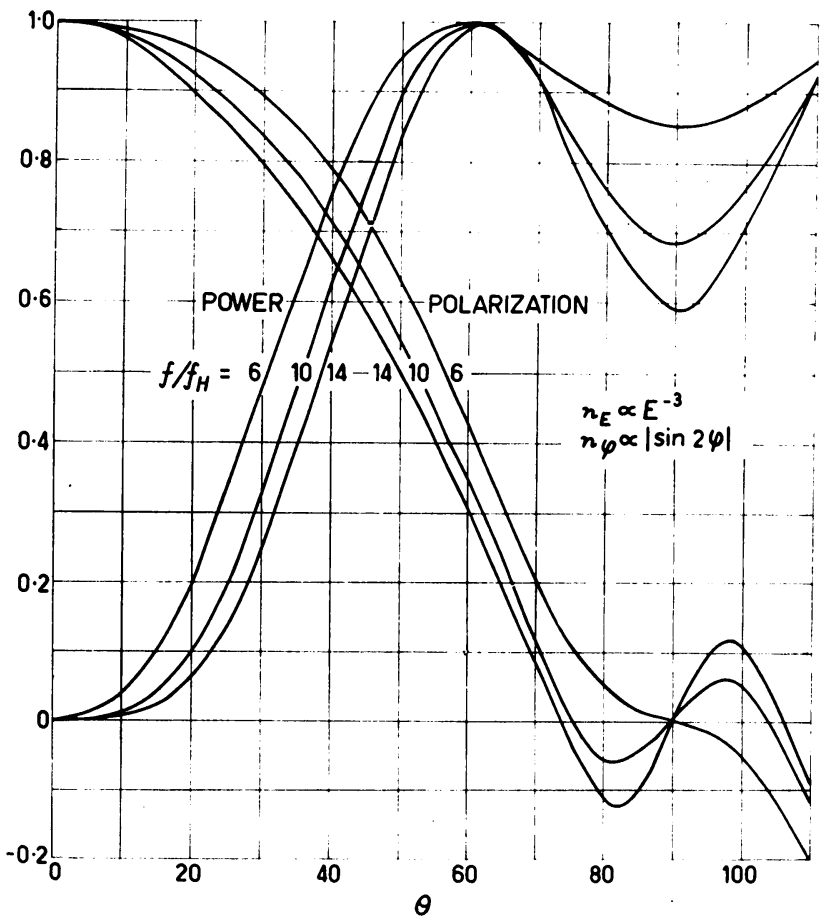

Fig. 10b.

Fig. 10a-b. The power and polarization polar diagrams of synchrotron radiation from mildly relativistic electrons with a powerlaw energy distribution and an (a) isotropic and (b) anisotropic pitchangle distributions. $\varphi$ represents the angle between the magnetic field direction and the observer (Dulk, 1970). 
The decrease in the magnetic field as the source moves outwards and expands causes the electrons to experience betatron deceleration. Since the time-scale of the field changes $\left(\sim 10^{2} \mathrm{~s}\right)$ is long compared with the gyro-period $\left(\sim 10^{-6} \mathrm{~s}\right)$, the magnetic moment remains adiabatically invariant and field changes are accompanied by energy changes such that $E_{\perp} / B=$ constant. The deceleration of the electrons is accompanied by a decrease in the emitted intensity, partly because as $f_{B}$ decreases, a higher harmonic is needed to radiate a given frequencly $f$, and partly because the number of electrons with $E>E_{0}$ decreases as $n_{E_{0}}(t)=[B(t)]^{\alpha}$. Also, since the deceleration affects only $E_{\perp}$, it decreases the number of electrons with large pitch angles $(\phi \approx \pi / 2)$. Electrons with $\phi \approx 0$ are also lost because they travel along magnetic field lines without mirroring. The anisotropic distribution $n_{\phi}=|\sin 2 \phi|$ is intended to simulate these conditions. These effects may partially explain the rapid fading and increasing polarization observed in the late stages of moving type IV sources.

\subsection{ASSOCIATION WITH OTHER METRE-WAVE CONTINUA}

We found in Section (2.1) that the fading of the moving type IV source is frequently accompanied by the brightening of one or two storm continuum sources (stationary type IV sources). Characteristically, the storm continuum is situated near the flare, possibly in a region of strong magnetic field. In terms of the above interpretation of the fading phase of a moving source, the storm-continuum source may sometimes be related to the 'dumping' of mildly-relativistic eletrons from the moving source.

We also noted in Section (2.1) that in some events the moving type IV burst was preceded by a stationary, unpolarized flare continuum. The onset of a striking example of a flare continuum above the limb, consisting of a rapidly expanding source, was attributed (Smerd, 1970) to the injection of fast electrons (possibly during the flash phase) into a bi-polar closed-field region. The mirroring of such particles can produce opposing electron streams in the trapping region which, in the manner described by Zheleznyakov and Zaitsev (1968) for type V bursts, emit unpolarized, second harmonic $\left(f \approx 2 f_{p}\right.$ ) radiation over an appreciable range of frequencies. The persistence of the flare continuum beyond several minutes may be due to repeated electron injections or to electron acceleration in the trapping region following the impact of the shock wave.

\subsection{ASSOCIATION WITH TYPE II BURSTS}

The present observations show a fairly strong association between moving type IV bursts and type II bursts. A case for a common shock wave as the source of both type II and moving type IV bursts has been made by Stewart et al. (1970) for three of the present events (1968 Oct. 23-4, Oct. 26; 1969 Mar. 21). The first of these, which we consider to show the closest relationship between the two bursts, has been described as Example 3 in Section 2.2.

Shock waves, not necessarily associated with type II bursts, have been involved as the sources of the moving bursts from the earliest interpretation (Boischot and Denisse, 1957). One moving type IV burst observed at Nançay with one-dimensional 
resolution at 169 and $408 \mathrm{MHz}$ (Boischot and Clavelier, 1967, 1968; Boischot and Daigne, 1968; Lacombe and Mangeney, 1969) was explained in terms of synchrotron radiation from electrons which were accelerated to relativistic energies within the advancing shock front. The explanation invoked a theory limited to weak (magnetic Mach number $M_{A} \lesssim 2$ ), collisionless shocks perpendicular to the magnetic field to provide a dissipative shock wave. The dissipative shock process provides the first stage of electron acceleration as had previously been suggested for type II bursts by Pikelner and Ginzburg (1963). Therefore, it seems that the physics of weak, collisionless, perpendicular plasma shocks - as understood at present - might account for the generation of both type II and type IV radiation by the same shock as it advances through the corona.

If we identify the disturbance with a weak, perpendicular shock moving at $M_{A}=v / v_{A}=2$ (where the Alfvén velocity $v_{A}=7 \times 10^{3} f_{B} / f_{p} \mathrm{~km} / \mathrm{s}$ ) then $v=2 v_{A}=$ $=1.4 \times 10^{4}\left(f_{B} / f_{p}\right)_{\mathrm{amb}}$ (where the subscript amb denotes the ambient medium through which the shock moves). In Section 2 we found that the synchrotron radiation of harmonic number $f / f_{B} \approx 10$ can best explain the burst characteristics. Therefore, for $80 \mathrm{MHz}$ radiation from inside the shock, we have $f_{B \text {, shock }} \approx 8 \mathrm{MHz}$ and $B \approx 3 \mathrm{G}$. We also found that the initial height of moving type IV bursts was near the $44 \mathrm{MHz}$ level in the standard model; we assume that this represents the enhanced plasma frequency within the shock, i.e., $f_{p \text {, shock }}=44 \mathrm{MHz}$. Using these values, together with the relation $\left(f_{B} / f_{p}\right)_{\text {shock }}=1.54\left(f_{B} / f_{p}\right)_{\text {amb }}\left(\right.$ Zaitsev, 1968), we find $\left(f_{B} / f_{p}\right)_{\text {shock }}=0.182,\left(f_{B} / f_{p}\right)_{\mathrm{amb}}=$ $=0.118$, and $v=1650 \mathrm{~km} / \mathrm{s}$.

This velocity is close to the observed velocity of the advancing front in Example 3. On the other hand, a shock moving at about $600 \mathrm{~km} / \mathrm{s}$ (the average source speed found earlier) is not likely to be a moving type IV source because high harmonic numbers $(\approx 30)$ are required. Also, a shock moving at, say, $3000 \mathrm{~km} / \mathrm{s}$ would require very low harmonic numbers $(\approx 5)$ and correspondingly high magnetic fields.

In the case of strong shocks $\left(M_{A} \gg 1\right)$ moving perpendicular to the magnetic field, we can use the relation $\left(f_{B} / f_{p}{ }^{2}\right)_{\text {shock }}=\left(f_{B} / f_{p}{ }^{2}\right)_{\text {amb }}$ (Helfer, 1953). The limiting value as $M_{A} \rightarrow \infty$ is $f_{p \text {, shock }}=2 f_{p \text {, amb }}$. Writing $v_{\text {shock }}=M_{A} v_{A}$ and substituting for $v_{A}$ in terms of $\left(f_{B} / f_{p}\right)_{\text {amb }}$ we obtain

$$
\left(f_{B} / f_{p}\right)_{\text {shock }}=\frac{v_{\text {shock }}(\mathrm{km} / \mathrm{s})}{3.5 \times 10^{3} M_{A}} .
$$

Choosing as before, $f_{p \text {, shock }}=44 \mathrm{MHz}$ and $\left(f / f_{B}\right)=10$, this gives $v_{\text {shock }}(\mathrm{km} / \mathrm{s})=$ $=1.27 \times 10^{3} \mathrm{M}_{A}$. Since, by definition, $M_{A} \gg 1$, this condition can only be satisfied for shock velocities that are faster $(>6000 \mathrm{~km} / \mathrm{s}$, say) than any source velocities yet observed. In the case of oblique shocks, only $B_{\perp}$ is involved in the jump conditions and the velocity of the strong shock is even higher.

On the other hand, Warwick $(1965,1968)$ identifies the moving type IV source with a disturbance moving parallel to the magnetic field and attributes the observed synchrotron radiation to relativistic electrons encountering the disturbance as they diffuse out along magnetic field lines from an accelerating region low in the Sun's 
atmosphere. He suggests that the disturbance, a large amplitude Alfvén wave or soliton, will degenerate into a shock as it moves in the corona; as such, it could also be a source of type II radiation. Longitudinal, collisionless shocks are known to exist, for example, in interplanetary space. Their large scale features have been successfully explained by aerodynamic methods (e.g. Sonnett, 1969); however, the wave-particle interactions that allow the wave to become a dissipative shock remain unspecified. Without this knowledge, we cannot discuss the radio emission from such waves.

These considerations lead us to conclude the moving type IV sources cannot always be identified with shock fronts of accompanying type II bursts. Does then the observed close association between type II and type IV bursts have some other physical significance? We suggest that an impinging shock front may be responsible for the expansion of a magnetic arch or that it may initiate or trigger the outward movement of the plasma-field configuration which is observed as a moving type IV source. We note that in such cases the speed and direction of the moving type IV source is not necessarily the same as that of the type II disturbance.

The almost invariable association of moving type IV bursts with active or disappearing filaments or prominences suggests that prominence material and magnetic field may be ejected from the low corona (perhaps by a shock wave) and later appear as a radio source. In some cases, the field structure may remain tied to the photosphere, resulting in an expanding arch. In other cases, magnetic reconnection may occur, resulting in a self-contained plasmoid which appears as an isolated source. In all cases, some of the material must be accelerated to relativistic energies to account for the observed radio emission.

\subsection{Physical Classification of the bursts}

We now attempt to use the results of the preceding discussion to provide a physical basis for the classification of the bursts as isolated sources, expanding arches, or advancing fronts.

The most clear-cut class is the expanding arch which, following Wild (1969), we identify with an expanding magnetic arch. It is clear that, depending on the orientation and location of such an arch on the Sun, the appearance of the arch as observed by the radioheliograph may differ considerably from event to event.

We identify the advancing front with the propagation through the corona of a MHD disturbance, either a soliton or a shock front. It is clear from example 3 of Section 2.2 that agreement between the type II speed (derived from the spectral drift rate) and the type IV speed (from projected positions) can be convincingly demonstrated from the observations only if the source movement is mainly transverse to the line of sight.

The isolated source illustrated by Example 1 was probably produced by solar ejecta in the form of a magnetized plasma cloud, part of the preceding eruptive prominence. A similar situation prevails in the event of 1969 March 1-2, the other 'long, distant and slow' event that seems to fit the same classification fairly unambiguously. However, it is important to recognize that in some circumstances an expanding arch 
or an advancing front might appear as an 'isolated source', e.g. an expanding arch seen edge-on or an advancing front intersecting a streamer structure.

In Table II we attempt to classify the 12 events into the proposed three types of moving-IV source.

TABLE II

Classification of 12 moving type IV bursts

Model A: Isolated source

1968 Feb. 25 (Models B and C cannot be ruled out because of short duration and small distance moved).

1968 Sept. 4 (Close relationship with type II burst leaves Model C possible).

1969 Mar. 1-2 (Large distance moved and source appearance strongly suggest this model).

1969 Oct. 10 (Advancing front intersecting a streamer structure, a variant of Model C, is possible). 1969 Dec. 17 (Two moving sources; peripheral movement of source 1 may fit variant of Model C). 1970 Mar. 21 (Large distance moved and source appearance suggest this model; same sense of polarization of all fragments may be more easily interpreted by Model B, expanding arch, seen edge-on). 1970 Apr. 29 (Appearance favours this model).

\section{Model B: Expanding magnetic arch}

1968 Oct. 26 (Strong polarization and jet-like appearance suggest arch seen edge-on).

1968 Nov. 22 (Appearance and polarization strongly suggest this model).

1969 Mar. 21 (Stewart and Sheridan's interpretation as an advancing front cannot be ruled out). 1969 Aug. 29-30 (Appearance and polarization support this model; advancing front cannot be ruled out).

\section{Model C: Advancing front}

1968 Oct. 23-24 (Source appearance and close relation with type II burst strongly suggest this model).

\section{Moving Type-IV Sources and Coronal Magnetic Fields}

\subsection{SOURCE MOVEMENT AND CORONAL FIELD}

In the case of an expanding magnetic arch the source movement and magnetic-field movement are inseparable. The magnetic loop links weak fields of opposite polarity in two regions as illustrated in Figure 11a. When the force which caused the expansion has spent itself the loop may contract to its original, much lower, position, it may remain as an 'exchange loop' at a high level (and may be carried out by the solar wind), or similar loops may be regenerated by photospheric currents.

The relation between an advancing front and the coronal magnetic field is more ambiguous. The 'slow' MHD wave (at $v \approx V_{T} \approx 170 \mathrm{~km} / \mathrm{s}$ ) is probably not involved in moving type IV bursts. Both the remaining magnetohydrodynamic wave modes propagate in the corona at about the Alfvén velocity; the Alfén wave propagates along the field only, the 'fast' wave in all directions. Meyer (1968) has found that the combined effects of the two wave modes make the direction of the magnetic field the preferred direction for coronal disturbances to propagate because it is the direction of least dispersion. Thus the advancing front type of moving type IV burst should move along the magnetic fields illustrated in Figure $11 \mathrm{~b}$. However, as noted in Section 3.4 shock-wave theories for type-II and moving type-IV bursts have been formulated only for perpendicular shocks. 


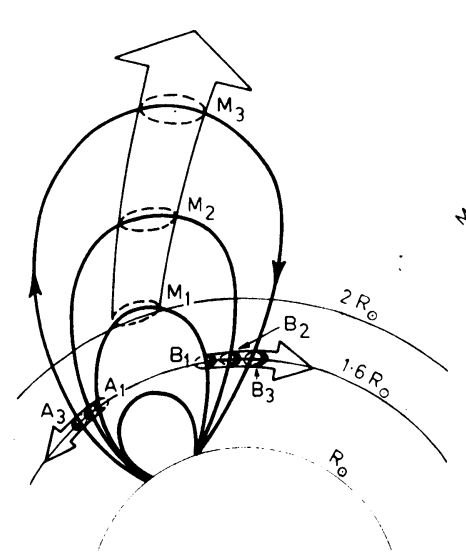

(a)

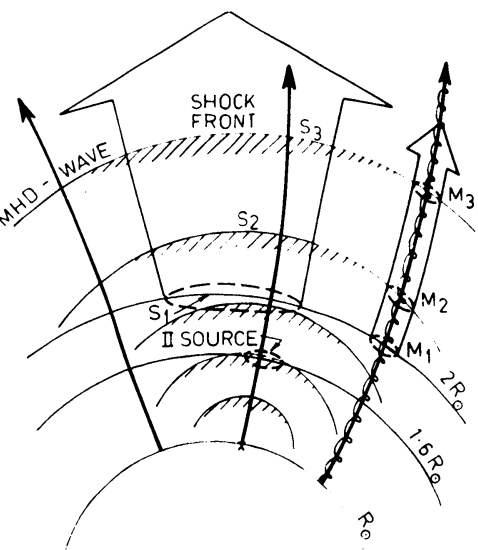

(b)

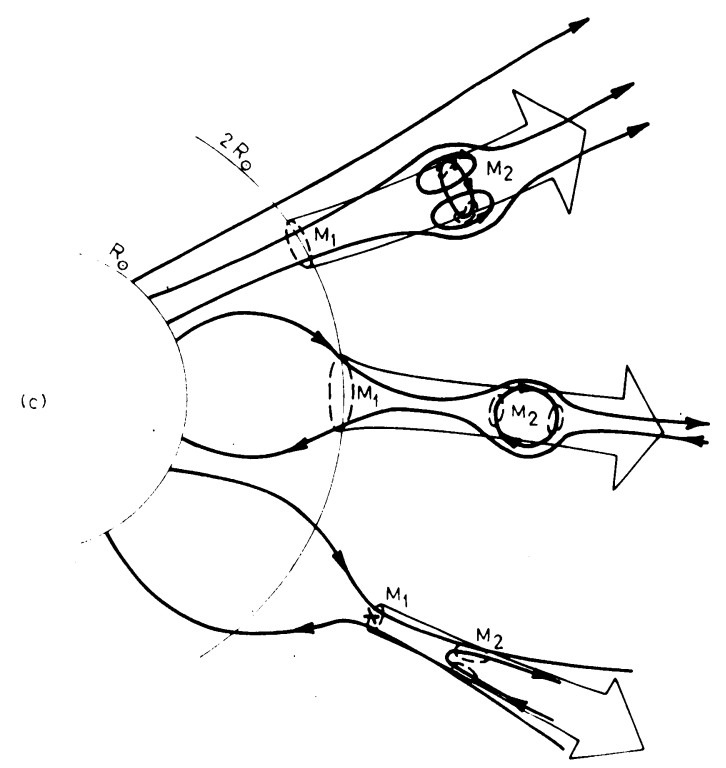

Fig. 11. Schematic diagrams of the magnetic-field structure in three models of moving type IV sources. The magnetic field lines are shown as thick arrowed lines, the paths of the moving IV sources by broad arrows. The optical disk, the $80 \mathrm{MHz}$ plasma level and the average starting height of the moving IV sources are shown by thin arcs at $R \odot, 1.6 R \odot$ and $2 R \odot$ respectively. The subscripts 1 , 2,3 , refer to the source positions at three successive times. (a) An 'expanding magnetic arch' with $A$ and $B$ representing plasma level sources and $M$ representing the moving source near the top of the arch. (b) An 'advancing front' of an MHD-wave moving along the magnetic field from an explosion near the surface. In the center and left, we depict a shock wave which generates type II radiation as it passes through the plasma level and synchrotron radiation beyond $2 R \odot$. On the right, relativistic electrons are shown traveling along the field line; they emit synchrotron radiation as they pass through the 'soliton' which propagates outwards at the much lower Alfvén velocity. (c) 'Isolated sources' moving out between open field lines. On top, the moving IV source is shown as a toroid with a poloidal stabilizing component moving in a unipolar field region. In the middle, the toroidal type IV source moves outwards between the opposing field lines of a helmet structure. At the bottom the moving IV source is at the end of an open-ended loop which retracts outwards after magnetic reconnection at $X$. 
In the case of isolated moving type IV sources due to ejecta from flare explosions, the motion, possibly at the Alfvén velocity, occurs along or between open field lines. Two open-line systems suggest themselves: those of a helmet structure where opposing field lines are separated by neutral sheets (see Figure $11 c$, middle), and the aligned 'radial' field lines high above unipolar regions (see Figure 11c, top).

A special case involving a helmet structure is Wild's (1970a) adaptation of Sturrock's (1966) model for the high-energy phase of a flare, illustrated in Figure 11c, bottom. The opposing fields, after reconnection across the neutral sheet, retract outwards and inwards from the place of reconnection. Wild suggests that the outwardmoving, open-ended loop may explain the isolated source in Exemple 1 and the other distant event of 1969 Mar. 1-2.

\subsection{Polarization AND SOURCE FIELdS}

In Section 3.2 we found that the general pattern of the evolution of moving type IV sources - decreasing intensity and increasing polarization - is compatible with the consequences of a gradual decrease of the magnetic field as the source moved outwards. We now discuss the observed polarization structure in relation to the magnetic field at the source and the wave-mode in which the radiation is emitted.

Wild (1969) has shown that the polarization structure on 1968 Nov. 22 is compatible with the emission of ordinary $(o)$ mode radiation from the two regions where the arch intersects the $80 \mathrm{MHz}$ plasma level and synchrotron radiation in the extraordinary $(x)$ mode from electrons at the apex of the arch, high above the plasma level. This situation is schematically illustrated in Figure $11 a$.

The same interpretation may apply to Example 4, the event of 1969 Mar. 21, and to the other events listed as possible expanding magnetic arches in Table II. The event of 1968 Oct. 26, when interpreted as an arch seen edge on, suggests that the field had a line-of-sight component towards the observer at the plasma level and away from the observer at the synchrotron heights. In the event of 1969 Aug. 29-30 the synchrotron source at the top of the arch showed both polarizations, implying a field component towards the observer on the west side and in the opposite direction on the east side of the arch. The plasma-level source on the west side of the arch was polarized and the one on the east side of the arch was unpolarized; this may indicate that the field on the east side was weak or that it was nearly perpendicular to the line of sight.

The polarization of the event of 1968 Oct. 23-24, the advancing front, was weakly LH all along the extended arc. For the $x$-mode to be LH the field must have a component away from the observer: the weak degree of polarization suggests that the field at the source was directed approximately $100^{\circ}-120^{\circ}$ from the observer. If the field lines were everywhere aligned along the advancing front, the line of sight component would necessarily reverse direction at some point along the arc. However, if the field lines were directed nearly radially inwards in the vicinity of the advancing front (which moved at an angle of approximately $60^{\circ}$ to the line of sight), the observed polarization could be explained. This suggests that the front advanced along the magnetic field. 
The remaining events in Table II are classified as isolated sources.

Polarization was not measured for the event of 1968 Feb. 25. For two events - 1968 Sept. 4, and 1970 Apr. 29 - the moving source remained single from beginning to end with the polarization increasing from $40 \%$ to $>85 \% \mathrm{LH}$ in the former and from $<10 \%$ to $>70 \% \mathrm{LH}$ in the latter event. The increasing polarization fits the pattern discussed in Section 3.2. Again, for $x$-mode radiation to be LH, the magnetic field within the source must have had a component away from the observer; the high degree of polarization makes it likely that at least late in the events, the sources contained few electrons in near-circular orbits and that the sources were observed at moderate angles $\left(\approx 20-40^{\circ}\right)$ with respect to the magnetic field.

In another event, that of 1969 Dec. 17, the two separate moving sources had different initial positions, different starting times, different speeds and moved in different directions. The first source moved peripherally and its polarization increased from 0 to $\approx 90 \% \mathrm{RH}$. Again we attribute the increasing polarization to the changing magnetic-field effects described in Section 3.2; the RH polarization suggests a magneticfield component towards the observer. The second moving source was strongly $\mathrm{LH}$ polarized from the beginning; the movement was approximately rectilinear until late in the event. The sense of polarization requires a magnetic-field component towards the observer. The high degree of polarization requires a deficiency of near circularorbit electrons throughout the event and a viewing angle of no more than $30^{\circ}$ with respect to the field.

The three remaining events - 1969 March 1-2, 1969 October 10 and 1970 March 21 - have intriguing polarization structures; the moving type IV phase began with a single source which later was accompanied by, or fragmented into, several moving sources.

On 1969 March 1-2 (Riddle, 1970) the initial source $A$, moving at $\approx 270 \mathrm{~km} / \mathrm{s}$ and unpolarized in the early stages, was joined after $\approx 1 \mathrm{hr}$ by another source $B$ about $11^{\prime}$ arc towards the NNE. Source $B$ faded from view after about $45 \mathrm{~m}$; source $A$ persisted for $40 \mathrm{~m}$ after $B$ had disappeared. The polarization of source $A$ started to increase $20 \mathrm{~m}$ before source $B$ appeared and reached $70 \% \mathrm{RH}$. The polarization of source $B$ increased, though not monotonically, from zero to $60 \% \mathrm{LH}$. Riddle suggested that the two sources formed part of the same structure, possibly ring-shaped, with a predominantly toroidal field stabilized by a poloidal component. Viewed side-on the two bright regions are those of greatest longitudinal extent near the extremities of the toroid where the field points towards and away from the observer respectively. This model could account for the observations with the field towards the observer at the leading edge. The model is illustrated at the top of Figure 11c, where the source is identified with an ejected 'plasmoid' moving out between the aligned open field lines above a unipolar region.

In the event of 1969 October 10 (Smerd, 1971), the original moving source $M_{1}$ fragmented into or was accompanied by source $M_{2}$ which lay about $12^{\prime}$ east of $M_{1}$; late in the event a third source $M_{3}$ appeared about $5^{\prime}$ east of $M_{1}$. The sources moved apart at about $300-400 \mathrm{~km} / \mathrm{s}$ while the centroid motion continued at $\approx 650 \mathrm{~km} / \mathrm{s}$. 
$M_{1}$ was initially unpolarized, but after fragmentation all sources became $\mathrm{LH}$ polarized. In the final, fading stages the polarization reached $\approx 50 \%$ for $M_{1}, \approx 30 \%$ for $M_{2}$ and $>80 \%$ for $M_{3}$.

The event of 1970 March 21 (Sheridan, 1970) was very similar in many respects. The initially single source, moving at $290 \mathrm{~km} / \mathrm{s}$, broke into 4 parts which moved apart at $\approx 90 \mathrm{~km} / \mathrm{s}$. The polarization increased from $30 \%$ to $>90 \%$, all parts having the same RH sense of circular polarization. Sheridan notes that a toroid cannot account for the observed polarization structure and considers Wild's (1970a) suggestion of an outward-moving, open-ended loop (the result of re-connection across a neutral sheet) illustrated at the bottom of Figure 11c. However, this model can produce multiple sources with like polarization only if viewed within a very small range of viewing angles near $90^{\circ}$, in which case the degree of polarization is low.

The results of Section 3.2 suggest that high polarization in several sources can be observed only if the field direction is about $20^{\circ}$ (or $160^{\circ}$ ) from the line of sight. One plasma-field configuration meeting these geometrical requirements is a twisted loop (like a Figure 8, though without intersecting lines) radiating at its extremities. Another possibility is a dipole field with a radiation belt. However, we know of no evidence that solar ejecta have such field configurations.

Wild (private communication) also suggested the model of an expanding magnetic arch seen nearly edge-on. We consider that this model provides the simplest explanation of the polarization of the events of 1969 Oct. 10 and 1970 March 21, providing that there is no reconnection of the dragged-out field lines, and providing that the breakup into several subsources can be explained.

\subsection{COMPARISON WITH CORONAL MAGNETIC-FIELD MAPS}

Altschuler and Newkirk (1969) described a technique for deriving the coronal magfield structure from Mount Wilson observations of the photospheric field. Using magnetometer observations taken at Mt. Wilson over a 27 day period, together with an approximate simulation of the effects of the solar wind, they calculate the threedimensional structure of the coronal field and display the results in stereo projection. As part of a joint investigation, they have produced coronal field maps pertaining to the times of several radioheliograph events (see also Newkirk, 1971). We now make a preliminary comparison of the radio burst positions observed in three events with the corresponding coronal field maps.

(1) Expanding arch of 1969 November 22 (Example 2 in Section 2.2). Figure 12 shows the expanding-arch positions at various times superimposed on one map of a stereo pair. The flare $X$ occurred underneath or at the edge of a low arch tunnel; the activated filament $Y$ lay at the far end of the tunnel. We suggest that the flare disturbance initiated an expansion of a portion of the magnetic arch tunnel. After the arch rose above the $80 \mathrm{MHz}$ level, the radio arch became visible. The polarization of the two halves of the arch in the early stages and the isolated 'feet' in later stages corresponds to the ordinary mode in the field configurations of the maps. This is consistent with the 

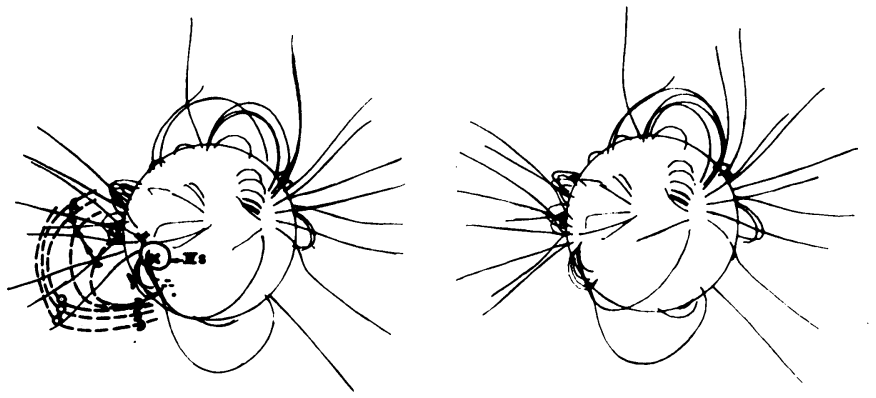

I968 NOVEMBER 22

Fig. 12. The 'expanding magnetic arch' of 1968 November 22 (Wild, 1969) plotted schematically on one of a stereo pair of coronal magnetic field maps (by courtesy of Newkirk and Altschuler, High Altitude Observatory, Boulder). The flare $(X)$ and active filament $(Y)$ seem to be located at the edge and at the far end of an arch tunnel respectively. The symbols (,), and $\bigcirc$ denote LH, RH, and unpolarized sources respectively. + and - magnetic source regions are denoted. The expanding arch traced out by the radio event is compatible with an expansion of a portion of the magnetic arch tunnel.

hypothesis that electron streams moving along the arch set up plasma oscillations and that the extraordinary mode radiation has difficulty in escaping (Wild, 1969). In later stages, the unpolarized central source presumably resulted from synchrotron emission from a concentration of electrons near the top of the arch. Finally, the location of the RH polarized storm continuum over a region of + field ( $\mathrm{N}$-polarity) implies that emission in the extraordinary mode is involved. This is opposite to the general rule, which implies ordinary-mode emission on the 'leading spot' hypothesis.

(2) Expanding arch or advancing front of 1969 March 21 (Example 4 in Section 2.2). Figure 13 shows the motion of the type IV burst super-imposed on the appropriate coronal field map. The flare $X$ and disappearing filament $Y$ occurred under high
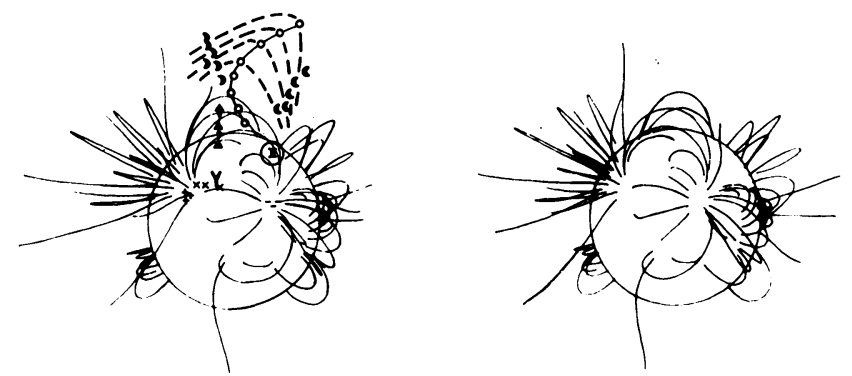

1969 MARCH 21

Fig. 13. The complex moving type IV event of 1969 March 21 plotted as in Figure 12. The flare $(X)$ and disappearing filament $(Y)$ seem to have occurred underneath high magnetic loops. If the event is interpreted as an 'expanding magnetic arch' it is found to be compatible with an expansion of these magnetic loops. 
magnetic loops. If, as suggested by Stewart and Sheridan (1970), the moving type IV burst was associated with an advancing shock front moving predominantly along the line of sight, then the maps give little information because no field lines are shown in this vicinity above about $1.5 R_{\odot}$. Alternately, we can interpret the event as an expanding arch as suggested earlier. In this case, the field maps relate closely to the type IV source. We suggest that the disturbance from the flare or disappearing filament caused the arch structure to move outward at an angle of $\approx 60^{\circ}$ to the line of sight. The radiation from the top of the magnetic arch is unpolarized; the regions on either side show opposite polarizations because of the oppositely directed magnetic-field components in the line of sight. The polarity of the radiation corresponds to the extraordinary mode in the field configuration of the maps. The polarization of the storm continuum source (possibly representing one foot of the arch), corresponds to the ordinary mode. Thus the polarization data and field maps are consistent with synchrotron emission from the moving source and with plasma radiation from the stationary source.

(3) Isolated source of 1969 March 1-2. Figure 14 shows the path of the isolated source of March 1-2 and the preceding eruptive prominence superimposed upon the corresponding coronal field map. Note that there were two oppositely polarized sources

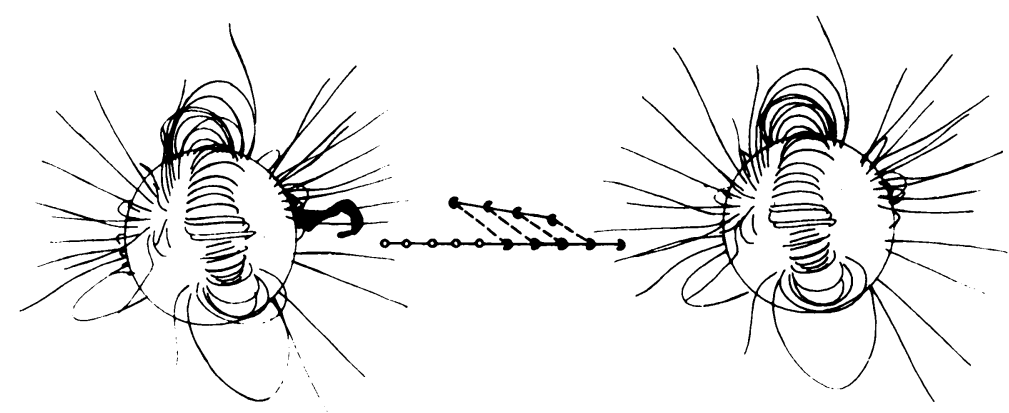

1969 MARCH 1-2

Fig. 14. The far-moving 'isolated source' type IV event of 1969 March 1-2 plotted as in Figure 12. The radio source seems to have moved out between open field lines of north (positive) polarity.

during the intermediate stages of the event. The coronal field lines in the region of interest are predominantly open, thus providing an open pathway for the moving source. The field lines are directed outwards, which results in $\mathrm{RH}$ polarization for the extraordinary mode. This agrees with the observed polarization of the dominant component of the burst. However, the LH polarization of the accompanying source, and probably the $\mathrm{RH}$ polarization of the dominant source as well, are presumably determined by the field structure carried out within the plasmoid. 


\section{References}

Altschuler, M. D. and Newkirk, G. Jr.: 1969, Solar Phys. 9, 131.

Boischot, A.: 1957, Compt. Rend. Acad. Sci. Paris 244, 1326.

Boischot, A. and Clavelier, B.: 1967, Astrophys. Letters 1, 7.

Boischot, A. and Clavelier, B.: 1968, Ann. Astrophys. 31, 445.

Boischot, A. and Daigne, G.: 1968, Ann. Astrophys. 31, 531.

Boischot, A. and Denisse, J. F.: 1957, Compt. Rend. Acad. Sci. Paris 245, 2194.

Dulk, G. A.: 1970, Proc. Astron. Soc. Austral. 1, 372.

Dulk, G. A.: 1971, Australian J. Phys. 24, 217.

Dulk, G. A. and Altschuler, M. D.: 1971, submitted to Solar Phys.

Dulk, G. A., Stewart, R. T., Black, H. C., and Johns, I. A.: 1971, Australian J. Phys. 24, 239.

Fung, P. C. W.: 1969, Can. J. Phys. 47, 179.

Ginzburg, V. L. and Syrovatskii, S. I.: 1965, Ann. Rev. Astron. Astrophys. 3, 297.

Helfer, H. L.: 1953, Astrophys. J. 117, 177.

Kai, K.: 1969a, Proc. Astron. Soc. Austral. $1,189$.

Kai, K.: 1969b, Solar Phys. 10, 460.

Kai, K.: 1970, Solar Phys. 11, 310.

Lacombe, C. and Mangeney, A.: 1969, Astron. Astrophys. 1, 325.

Meyer, F.: 1968, in K. O. Kiepenheuer (ed.), 'Structure and Development of Solar Active Regions', IAU Symp. 35, 485.

Newkirk, G. Jr.: 1961, Astrophys. J. 133, 983.

Newkirk, G. Jr.: 1971, this volume, p. 547.

Pikelner,S. B. and Gintsburg, M. A. : 1963, Astron. Zh. 40, 842 = Sov. Astron. 7, 639, 1964.

Riddle, A. C.: 1970, Solar Phys. 13, 448.

Sheridan, K. V.: 1970, Proc. Astron. Soc. Austral. 1, 376.

Smerd, S. F.: 1970, Proc. Astron. Soc. Austral. 1, 305.

Smerd, S. F.: 1971, Australian J. Phys. 24, 229.

Sonett, C. P.: 1969, Comments Astrophys. Space Phys. 1, 178.

Stewart, R. T.: 1971, Australian J. Phys. 24, 209.

Stewart, R. T. and Sheridan, K. V.: 1970, Solar Phys. 12, 229.

Stewart, R. T., Sheridan, K. V., and Kai, K.: 1970, Proc. Astron. Soc. Austral. 1, 313.

Sturrock P. A.: 1966, Nature 211, 695.

Van de Hulst, H. C.: 1950, Bull. Astron. Inst. Neth. 11, 135.

Warwick, J. W.: 1965, in Solar System Radio Astronomy, ed. by J. Aarons, p. 131, Plenum Press, N.Y. Warwick, J. W.: 1968, Solar Phys. 5, 111.

Weiss, A. A.: 1963, Australian J. Phys. 16, 526.

Wild, J. P.: 1969, Solar Phys. 9, 260.

Wild, J. P.: 1970a, Proc. Astron. Soc. Austral. 1, 365.

Wild, J. P.: 1970b, Proc. Astron. Soc. Austral. 1, 348.

Wild, J. P., Sheridan, K. V., and Kai, K.: 1968, Nature 218, 536.

Wild, J. P., Sheridan, K. V., and Trent, G. H.: 1959, in Paris Symp. Radio Astron. (ed. by R. N. Bracewell), p. 176, Stanford University Press.

Zaitsev, V. V.: 1968, Astron. Zh. 45, 766; 1969, Sov. Astron. 12, 610.

Zheleznyakov, V. V. and Trakhtengerts, V. Yu.: 1965, Astron. Zh. 42, $1005=$ Sov. Astron. 9, 775, 1966.

Zheleznyakov, V. V. and Zaitsev, V. V.: 1968, Astron. Zh. 45, 19; 1968, Sov. Astron. - A. J. $12,14$. 